\title{
¿Deberíamos dejar de hacer angioplastia renal? Estudio CORAL
}

\author{
Should we stop making renal angioplasty? CORAL trial
}

La causa predominante de estenosis de la arteria renal es la aterosclerosis ${ }^{1}$ y probablemente su revascularización sea uno de los temas más controvertidos dentro del intervencionismo endovascular.

Estudios observacionales y registros previos han mostrado beneficios de la angioplastia en relación a la función renal y al mejor control de la hipertensión arterial, ${ }^{2}$ pero estas observaciones no fueron confirmadas por los estudios aleatorizados, a los cuales se les adjudicaba serios errores de diseño. ${ }^{3}$ Además, la eficacia clínica para reducir eventos cardiovasculares, por efecto indirecto de la revascularización, era incierta. Por ello, el estudio CORAL (Cardiovascular Outcomes in Renal Atherosclerotic Lesions), ${ }^{4}$ recientemente publicado, fue diseñado para mitigar estas deficiencias de los estudios anteriores.

De 5322 pacientes analizados, ingresaron al estudio 947. El criterio de admisión fue tener estenosis aterosclerótica de la arteria renal e hipertensión arterial sistólica (con necesidad de dos o más drogas) y/o disfunción renal. Fueron aleatorizados a la rama de tratamiento médico más angioplastia con stent 467 pacientes y a tratamiento médico contemporáneo 480 (incluye un bloqueante de los receptores de angiotensina con o sin diuréticos tiazídicos, amlodipina, terapia antiplaquetaria, atorvastatina y manejo de la diabetes según las guías actuales).

El objetivo primario fue una combinación de muerte de causa cardiovascular o renal, infarto de miocardio, accidente cerebrovascular, hospitalización por insuficiencia cardíaca, insuficiencia renal progresiva y/o necesidad de diálisis. Los dos grupos tenían características basales similares. Al seguimiento, con una mediana de 43 meses, no se observaron diferencias significativas en el punto final compuesto primario entre ambas ramas (angioplastia 35,1\% vs. tratamiento médico 35,8\%; $\mathrm{p}=0.58$ ) ni tampoco considerando por separado cada componente. Ambos grupos aumentaron el número de medicamentos administrados y sí se observó una modesta pero significativa reducción de la presión arterial sistólica con la angioplastia (tratamiento médico $-2,3 \mathrm{mmHg} v$ s. angioplastia $-4,4 \mathrm{mmHg} ; \mathrm{p}=0,03$ ). Los autores concluyen que la angioplastia de la arteria renal, comparada con el tratamiento médico contemporáneo, no otorga beneficios en término de eventos clínicos en pacientes con estenosis de la arteria renal e hipertensión arterial y/o disfunción renal.

Si bien es cierto que el estudio CORAL fue mucho más prolijo en su diseño que estudios anteriores, hay varias consideraciones que podríamos hacer.

En primer lugar, el número programado y estadísticamente necesario de participantes (1080) no se cumplió, "ingresaron un número cercano, 947 pacientes", debido al lento reclutamiento.

Segundo, el grado de hipertensión arterial es un importante marcador de actividad neuroendocrina y severidad fisiológica de la estenosis renal, por lo que, al reclutar pacientes con HTA y buena respuesta al tratamiento médico, no deberíamos esperar que la revascularización tenga un claro beneficio, y si la disfunción renal está presente, podría ser de causa parenquimatosa. Por eso, determinar el índice de resistencia intraparenquimatosa renal (no valorado en este estudio) podría ser importante, pues marca el grado de nefroangioesclerosis, descripta como importante predictor de éxito de la revascularización. ${ }^{5,6}$ Aunque un índice de resistencia (IR) $\geq 0,80$ no puede ser recomendado como parámetro predictivo del resultado de la intervención, ${ }^{7}$ un IR bajo $(<0,80)$ indica una mayor probabilidad de que el paciente se beneficie de la revascularización. ${ }^{8}$

Tercero, determinar y definir cuál sería el grado de estenosis que justifique la revascularización también es importante. En modelos experimentales, la estenosis renal $>75 \%$ empieza a generar gradientes de presión translesional y sólo estenosis $>80 \%$ del diámetro del vaso producen un cambio del metabolismo aeróbico a anaeróbico en la células tubulares y caída de la función renal; ' a pesar de ello, aún no ha sido bien definida la condición de nefropatía isquémica. Con estas observaciones podríamos decir que los criterios de inclusión anatómicos del CORAL tienen pobre relevancia fisiológica, ya que los hallazgos angiográficos determinaron una estenosis renal del $67 \%$, valor inferior a lo considerado como significativa.

Cuarto, los métodos diagnósticos no invasivos tienen sus limitaciones y la falta de correlación entre la severidad angiográfica con los gradientes hemodinámicos permitió, probablemente, el ingreso al estudio de un 
gran número de pacientes con hipertensión esencial y estenosis de la arteria renal no significativa, en donde podría esperarse la falta de beneficio con la angioplastia renal. Apoya esta afirmación el hecho de que el número de medicamentos antihipertensivos administrados aumentó de 2 a cerca de 3 en ambos grupos y que la reducción de la presión arterial era muy similar. En el futuro, el único criterio de ingreso debiera ser invasivo, angiografía con varias proyecciones y determinación del gradiente transestenótico.

Quinto, para valorar el impacto de la revascularización sobre la función renal, se debería considerar previamente (año/años antes de la inclusión) el grado de progresión de la disfunción renal y no un valor aislado previo; con este análisis uno podría determinar si la revascularización modificó o no el grado de progresión y averiguar si los pacientes con deterioro más o menos rápido de la función renal basal (antes de la inclusión) se benefician de una intervención al modificar la curva de deterioro funcional.

Sexto, al seguimiento, con una mediana de 43 meses y al analizar las curvas de eventos, se puede observar que comienzan a separarse a partir del tercero al cuarto año, aunque no significativamente (probablemente por el bajo número de pacientes), por lo que cabría preguntarse si el seguimiento no fue demasiado corto, especialmente teniendo en cuenta que la enfermedad aterosclerótica renal es progresiva ${ }^{10} \mathrm{y}$ se tomaron puntos finales clínicos.

Este ensayo muestra que en los pacientes que tienen hipertensión arterial y estenosis renal moderada a severa de la arteria renal, la terapia médica contemporánea fue tan eficaz como la angioplastia renal ya que la terapia médica sola tuvo un $20 \%$ de punto final primario a dos años, la mitad de la esperada (40\%). ${ }^{11}$ Esto sugiere que las drogas usadas (candesartán, diuréticos tiazídicos, amlodipina y atorvastaina) fueron beneficiosas. El estudio CORAL confirma los hallazgos del estudio ASTRAL. ${ }^{12}$ Sin embargo, la discordancia entre la alta tasa de éxito de la angioplastia y la baja o nula respuesta clínica probablemente se deba a la pobre selección de pacientes, mala discriminación de la severidad de la estenosis y a la presencia concomitante de enfermedad renal parenquimatosa.

Personalmente, creo que la angioplastia renal sigue teniendo un rol importante, ya que con cierta frecuencia hemos observado pacientes en los que la revascularización fue efectiva, especialmente aquellos con edema agudo de pulmón súbito y estenosis renal severa,y pacientes monorrenos o con estenosis críticas de ambas arterias renales (isquemia global). La revascularización también debería estar reservada para pacientes que no responden al tratamiento médico antihipertensivo o que no lo toleran y tienen una lesión crítica de las arterias renales. Por ello, la confirmación invasiva de la significancia funcional es esencial. El desafío actual es individualizar cuáles son los pacientes que se beneficiarán de la angioplastia frecuentemente excluidos de los estudios.

Palabras clave: estenosis de arteria renal, aterosclerosis, angioplastia renal, estudios aleatorizados.

\author{
Dr. Arturo J. Fernández Murga \\ Instituto de Cardiología de Tucumán - Clínica Mayo Tucumán. \\ Av Mitre 760, (4000) San Miguel de Tucumán. Argentina | Tel/Fax: 0381-4330389| fdezmurga@tucbbs.com.ar
}

\title{
BIBLIOGRAFÍA
}

1. TextorSC, Lerman L. Renovascular hypertension and ischemic nephopathy. Am J Hypertns 2010;23:1159-1169.

2. Leertouwer TC, Gussenhoven EJ, Bosch JL, et al. Stent placement for renal arterial stenosis: where do we stand?. A meta-analysis. Radiology 2000;216:78-85.

3. Fernández Murga AJ. Análisis Críticos de los estudios sobre angioplastia renal. Rev Arg de Cardioangiología Intervencionista (RACI) 2013;4:220-225.

4. Cooper CJ, Murphy TP, Cutlip DE, et al. Stenting and medical therapy for atherosclerotic renal-artery stenosis (CORAL Trial). N Engl I Med 2014;370:13-22.

5. Radermacher J, Chavan A, Bleck J, et al. Use of Doppler ultrasonography to predict the outcome of therapy for renal-artery stenosis. N Engl I Med 2001;344(6):410-417.

6. RundbackJH, Sacks D, Kent C, et al. Guidelines for the reporting of renal artery revascularization in clinical trials. J Vasc Interv Radiol 2000;23:959-974.

7. Krumme B, Hollenbeck M. Doppler sonography in renal artery stenosis - does the Resistive Index predict the success of Intervention? Nephrol Dial Transplant 2006. Editorial comment.

8. Hoffman O, Carreres T, Sapoval MR, et al. Ostial renal artery stenosis angioplasty: inmediate and mid-term angiographic and clinical results. J Vasc Interv Radiol 1998;9:65-73.

9. Imanishi M, Akabanes, Takamiya M, et al. Critical degree of renal arteria stenosis that causes hypertension in dogs. Angiology 1992;43:833-842.

10. Safian R, Textor S. Renal artery stenosis. N Engl J Med 2001;344:431-442. Review article.

11. Cooper CJ, Murphy TP, Matsumoto A, et al. Stent revascularization for the prevention of cardiovascular and renal events among patients with renal artery stenosis and systolic hypertension: rationale and design of the $\mathrm{CO}$ RAL trial. Am Heart J 2006; 152:59-66.

12. The ASTRAL Investigators. Revascularization versus medical therapy for renal-artery stenosis. N Engl J Med 2009;361:1953-1962. 